\title{
EDITORIAL
}

\section{Aldosterone 'escape' vs 'breakthrough'}

S everal recent publications have used the terms 'aldosterone escape' and 'aldosterone breakthrough' interchangeably. This tendency obscures the pathophysiology of these different processes, both of which have very important clinical implications. Administration of large doses of aldosterone typically causes an initial decrease in urinary sodium excretion, which leads to renal sodium retention. However, urinary sodium excretion subsequently increases to balance sodium intake before detectable edema develops. This phenomenon, termed 'aldosterone escape', is the reason why edema formation is not a characteristic of primary hyperaldosteronism. The process of aldosterone escape invokes several mechanisms. In addition to increasing renal perfusion pressure, the resultant volume expansion decreases proximal sodium reabsorption and increases sodium delivery to the distal nephron sites of mineralocorticoid action. This increased delivery of sodium overrides the enhanced aldosterone sodium reabsorption at the site of mineralocorticoid action. Moreover, volume expansion, secondary to the action of aldosterone, increases levels of plasma natriuretic hormone and therefore its inhibitory effect on sodium reabsorption in the collecting duct. Together, these events contribute to normal aldosterone escape and the prevention of edema.

In edematous disorders including cardiac failure and cirrhosis, however, normal aldosterone escape is impaired. Underfilling of the arterial circulation in cardiac failure secondary to decreased cardiac output or primary systemic arterial vasodilation in cirrhosis activates the neurohumoral axis, stimulating the reninangiotensin-aldosterone system (RAAS) and the sympathetic nervous system. In this setting, the decrease in renal perfusion pressure, the decline in glomerular filtration rate, and the increase in proximal tubule reabsorption diminish the distal delivery of sodium to the sites of aldosterone and natriuretic hormone action in the collecting ducts. These processes lead to impaired aldosterone escape and blunt the actions of natriuretic hormone, resulting in edema formation (Schrier, R. W. Renal and Electrolyte Disorders $7^{\text {th }}$ edn 45-85 [Lippincott Williams \& Wilkins, Philadelphia, 2010]).

The aldosterone breakthrough phenomenon describes a totally different process from aldosterone escape. Recognition of the circulating and intrarenal role of the
RAAS in various disease states, including hypertension and the edematous disorders, led to the development of angiotensin converting enzyme (ACE) inhibitors that decrease levels of circulating angiotensin II and aldosterone. The angiotensin receptor blockers (ARBs) were subsequently developed, which inhibit the action of type 1 angiotensin II receptors with a resultant decrease in aldosterone level. ACE inhibitors and ARBs are now widely used with beneficial effects, particularly in patients with hypertension and cardiac failure. However, since angiotensin II inhibits renin release, a large increase in plasma renin activity occurs with the administration of either ACE inhibitors or ARBs. Moreover, after several weeks of ACE inhibition or ARB therapy, plasma aldosterone returns to pretreatment levels in up to $30-40 \%$ of patients. This phenomenon has been termed aldosterone breakthrough (Bomback, A. \& Klemmer, P. Nat. Clin. Pract. Nephrol. 3, 486-492; 2007). Reports that those patients who demonstrate aldosterone breakthrough have a worse clinical prognosis than those who do not demonstrate aldosterone breakthrough also exist. This deleterious effect could be due to the nongenomic effects of aldosterone, which are known to cause inflammation, fibrosis and oxidant injury. Whether addition of a mineralocorticoid antagonist to the treatment regimen would ameliorate the proposed negative clinical consequences of aldosterone breakthrough in these patients is not known.

Of note, direct renin inhibition, for instance with aliskiren, does not increase plasma renin activity, and therefore may not be associated with aldosterone breakthrough. Direct renin inhibitors affect the enzymatic action of renin, but not its production or interaction with the renin receptor. The effect of renin on the renin receptor has been proposed to be profibrotic (Nguyen, G. et al. J. Clin. Invest. 109, 1417-1427; 2002). Of further interest, vitamin $\mathrm{D}$ has been shown to bind to the promoter region of the renin gene and downregulate renal messenger RNA expression of renin (Doorenbos, C. R. C. et al. Nat. Rev. Nephrol. 5, 691-700; 2009). Therefore, direct renin inhibitors, vitamin D or a combination of the two could theoretically exert a renal and/or cardiovascular protective effect in patients receiving ACE inhibitor or ARB treatment who demonstrate aldosterone breakthrough.

doi:10.1038/nrneph.2009.228

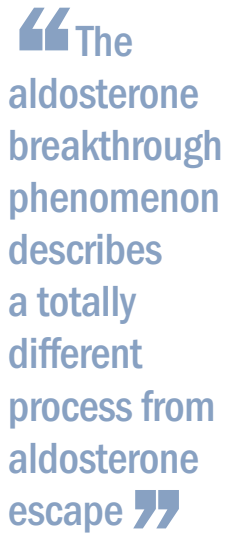

Robert W. Schrier is the Editor-in-Chief of Nature Reviews Nephrology.

Competing interests The author declares no competing interests. 